\title{
Obtaining the Optimum Block Length of the Chet Network: An At-Grade Transportation Network without Signalized Intersections, Roundabouts, or Stop Signs
}

\section{S. P. Liyanage and S. Pravinvongvuth}

\begin{abstract}
A typical at-grade transportation network forms a grid consisting of intersections. The majority of delays and accidents in the network are caused by traffic movements through those intersections. Even though the use of controlling systems gets rid of conflict movements at intersections, it increases the congestion due to capacity reduction. To overcome this important issue, designing zeroconflict transportation network is essential. A novel design of an at-grade transportation network without signalized intersections, roundabouts, or stop signs was proposed. It is called the Chet network, which can be used as an alternative form of urban streets for a built environment. Within the Chet network, a car can move from one place to another without facing any conflict movement at any junction while still maintain the low cost of at-grade infrastructure. The network is composed of hexagon blocks tiling together with a unique arrangement of one-way or two-way directional links to avoid conflict movements at all junctions. This study aims further to explore the concept of the Chet network by constructing several testing cases in microscopic traffic simulation to obtain the optimum block length in forming hexagons in the Chet network, which is an important step in moving forward to implementation in real-life.
\end{abstract}

Keywords: Hexagonal network topology; Traffic flow analysis; Transport dynamics; Simulation of traffic system; Network design

\section{Introduction}

The topology of an urban street network is crucial in developing a city. Grammenos et al. [13] stated, properly designed street patterns lead to healthy, peaceful and safe surroundings that immensely contribute to the quality of life and sustainability of the environment. The grid network is the most widely used topology of urban street networks worldwide over the past centuries. As stated by Lewis Mumford, "Trend is not destiny" and he highlights that reviewing this long-lasting urban street and land use pattern to verify whether it is the most efficient topology yet is important.

Even though a rectangular grid of urban streets is simple and incredibly easy to plan or build, it has many major disadvantages. It does not allow diagonal movements as stated by Prince A. [24]. which can be a shortcut in traveling. According to Bally D. [1], transportation and urban infrastructure planners, sociologists, environmentalists, geographers and architects have mentioned avoiding grid patterns should be done in practices. In Cauchon's words [4, 5, 6], grid network "is not only ugly, dangerous and unhealthy, but also inefficient and expensive". Therefore, whether the existing grid network is the ideal transportation network to accommodate current vehicle demand is questionable. However, it is still the most popular urban street network in the practice.

A typical grid network requires intersections when two roads are crossing. Within each intersection, there are 12 possible turns ( 3 turns per each of the 4 approaches) causing 32 conflict points. Among those, there are 16 primary (crossing), 8 secondary (merging) and 8 minor (diverging) conflict points.

This highlights the importance of reducing the risk of accidents and enhancing safety by avoiding conflict movements at intersections. Even though transportation engineers commonly use controlling systems such as traffic signals, stop signs, roundabouts, right turn prohibitions (US traffic) and one-way streets to reduce those conflict movements, none

Eng. (Ms.) S. P. Liyanage, AMIE(SL), B.Sc. Eng. (Hons) (Asian Institute of Technology), M. Eng. (Asian Institute of Technology), Ph.D. student, Faculty of Science, Engineering, and Technology, Swinburne University of Technology.

Dr. S. Pravinvongvuth, B. Eng. (Hons) (Chulalongkorn University), M. Eng. (Asian Institute of Technology), Ph.D. (Utah State University, USA), Assistant Professor, Faculty of Transportation Engineering, Asian Institute of Technology 
of them can completely get rid of accidents but evidently cause delays to the traffic. The most widely used intersection controlling system is stop signs for the light traffic and traffic signals for the medium or heavy traffic as stated in FHWA [11]. A roundabout can also be used at an intersection to remove primary conflict points, even though it is not considered as a traffic control device. Roundabouts are less popular and usually installed at isolated intersections where the traffic volume is low. Roundabouts are not capable of accommodating high demands due to short-distance weaving within it. In addition, it requires a larger space than a typical intersection. These intersection control systems increase the safety at every intersection all over the world by reducing conflict points, yet add a cost of additional travel time. When passing a certain intersection, most of the time, vehicles must stop to avoid conflict. When the amount of traffic is high, gridlock could happen and cause significant delay along the entire network. Another option for the at-grade intersection conflicts is grade-separated interchanges. Even though it is better in terms of safety, major drawbacks exist, as it is expensive and land consuming. In addition, if the demand is high, the queue spillback from the next intersection or merging at the bridge could cause congestion anyhow. If transportation planners could develop such a network that has no intersection delay and ensure safety, it would be wonderful.

The use of stop signs might be the worst in terms of delay because every vehicle must stop even when there is no vehicle from any conflict turn. Hence, this is not popular in high demand situations. Comparatively, the use of traffic signals could better but as Pravinvongvuth S. [23] explained, the chance that a vehicle can move through an intersection without being stopped is $50 \%$ for an intersection under twophase traffic signals (50-50 time split) when permitted left turn (US traffic) is used. This is when ignoring the start-up loss time, all-red time or four phase signal timing plans. Therefore, when the above-mentioned delays are present, chances will be even lesser than $50 \%$.

This proves the deficiency of the long lasted rectangular grid. The necessity of thinking outside the grid is needed to develop an at-grade transportation network topology where vehicles can move from one end to another without stopping. The initiative in proposing better alternatives as a replacement for the rectangular street grid begun in the early 1900s. Alternatives include hexagonal street patterns and other patterns engaged with loops, cul-de-sacs, and curvilinear roads.

Another solution for intersection delay is signal coordination, even though it results in higher capacities but still outlying the ideal capacity. In any kind of signal coordination, conflict movements are not removed but separated in time by traffic signals as stated in Wong [25]; Pillai et al. [22]; Girianna and Benekohal [12]; Dotoli et al. [7]; Fan [9]; Han and Gayah [15]. Vehicles on the coordinated street gain benefit mainly on travel times, while other streets are worse off. Recently, Eichler et al. [8] proposed a novel approach to reducing conflict movements in a typical grid network by rearranging link directions. However, that design failed to remove conflict movements completely. Even though their vortex-based design can remove primary (crossing) conflicts, secondary (merging) and minor (diverging) conflicts still exist. The existence of minor conflicts does not significantly affect the performance of a road network, yet, secondary conflicts do. They may cause a delay or a risk of having an accident. This design has another drawback; i.e., it increases average travel distances when comparing to unrestricted conflicted or intersected flow. Therefore, it is important to highlight that the above-mentioned approaches (signal coordination, roundabout, and the vortex-based design) are remedies to the existing problematic grid intersections. However, since they are based on the grid network, problems of at-grade intersections are unavoidable. To, completely, remove all conflict movements at intersections, we must think out of the grid. Exploring an alternative design of at-grade transportation network is essential.

\section{Literature Review}

In the early 1900s, there were two alternatives proposed as a better replacement for the rectangular street grid. They were hexagonal and other street patterns, which are engaged with loops, cul-de-sacs, and curvilinear roads. Afterward, American land use developers propagated the cul-de-sac design as the ideal pattern, which concealed further researching on hexagonal street patterns as per Bally D. [1].

Tiling a plan can be done by using one of the three regular polygons; triangles, squares and, hexagons. Even though Melut [18] proved that the triangle is the best among those three polygons in terms of transportation cost, they are not suitable to be used in transportation 
networks, as the turning angle at an intersection is only 60 degrees. The grid, which is used over the centuries, consists of rectangles or squares with 90-degree angles. Use of hexagons as a replacement for squares or rectangles is under research as it is becoming interesting to explore as it accounts for most of the problems of the existing rectangular street grid.

When considering the appropriate block pattern for the street network, the economy of a road should also be considered as the impacts of saving also includes infrastructure and utilities such as electricity, water, sewer, etc. As Hales [14] stated, unlike any other patterns, hexagons provide the highest area with the smallest perimeter. Therefore, the use of hexagons is optimal in terms of economy. With the assumption that average distance to the network is same or standardized, construction and maintenance cost will be same disregarding which regular polygon (triangle, square or rectangle, hexagon) is used as the network block as stated by Melut [18]. In case of symmetry, rectangular networks are better as hexagonal network with an even number of blocks lack symmetry. Nevertheless, when comparing the hexagonal network with the rectangular grid, the hexagonal network provides a minimum perimeter for fixed areas and maximum areas for fixed perimeters.

However, few works of literature, have proposed the idea of using hexagons in the transportation network, those are Ben-Joseph and Gordon [2]; Bally [1]; Omolo-Okalebo and Sengendo [20]. However, none of them accounts for the perspective of transportation: how to arrange link directions to avoid conflict movements at junctions. Therefore, Pravinvongvuth. S. [23] has taken the initiative to propose a new idea for at-grade transportation network design. This is achieved through the unique arrangement of one-way and two-way links towards forming hexagons tiling to avoid conflict movements at junctions. Since there is no conflict movement at any junction, there is no need of any intersection control. In this regard, vehicles can move from one point to another without stopping. This significantly reduces intersection delay and conflicts to enhance safety in transportation.

\section{The Chet Network}

As shown in Figure 1, one-way and two-way links in the Chet network are uniquely arranged in forming congruent hexagonal blocks where vehicles can move from one place to another without facing any conflict movement at intersections. Consider the US traffic system where the vehicles move on their right. As shown in Figure 1, each one-way link represents two-lane one-direction roads while each twoway link represents two-lane roads (one-lane per each direction). There are two types of hexagons: (1) clockwise and (2) counterclockwise traffic circulation within the block. Each counterclockwise hexagon is surrounded by six clockwise hexagons. No counterclockwise hexagon is placed adjacent to another; there must be clockwise hexagons in between. This is the uniqueness in the design of the Chet network design. Each hexagon (block) represents a land area either used commercially (attracting traffic in the morning) or residentially (generating traffic in the morning). More precisely the commercial land area can be any land use where vehicle trips are attracted to in the morning while the residential land area can be any land use where the vehicle trips are generated from in the morning. In the illustrative network, each counterclockwise traffic circulated block is a commercial zone, while each of the sixclockwise traffic circulated blocks surrounding the counterclockwise hexagon are residential zones. The reason is a commercial zone typically denser than a residential zone; however, that is not a limitation of the network. Mixed land uses can also be considered.

The Chet network is composed of concurrent hexagons tiling together and the main reason for this specific and unique design arrangement of one-way/two-way links is to avoid conflict movements at junctions as proposed by Dr. Surachet Pravinvongvuth as an alternative form of an at-grade transportation network. Hence, there is no need to have signal controllers. However, minor delays such as lane changing delays still exist as in other transportation networks.

Moreover, it is important to highlight the junction design of the proposed network by zooming in on one of the junctions of the network. At each junction of the Chet network, there are two one-way links and one two-way link. A vehicle that comes to a junction will have only two options, either turning slight left or slightly right. The slight turn here is 120 degrees, which is advantageous relative to the 90-degree turn of the grid network. Omolo-Okalebo and Sengendo [20] noted that hexagonal city form was proposed as the most optimum way of land usage as Y-shaped roads are better suited to motor traffic than conventional crossroads. The most prominent characteristic of the proposed 
junction design is not having any conflict movement. Hence, there is no need to install any intersection control while the network still maintains the least cost of at-grade (no over or underpass). Even without intersection control, the network has the potential to minimize the total delay. That is because, in most (if not all) networks, most delays happen at intersections. Each block here serves as a roundabout, but with much longer weaving sections.

This observation, however, leads to the need for network evaluation at the microscopic level. The initial study of this new at-grade hexagonal transportation network without any signal control has been originally designed and compared the performance with an equivalent existing street grid network as in the Pravinvongvuth, S. [23]. In the initial study, a performance analysis of the typical grid network and that of the proposed Chet network were compared by considering several testing cases using a microscopic traffic simulator. The typical rectangular grid was equivalently reformulated using the Chet network structure by considering equivalent size, equivalent land use density, and total network travel demands.

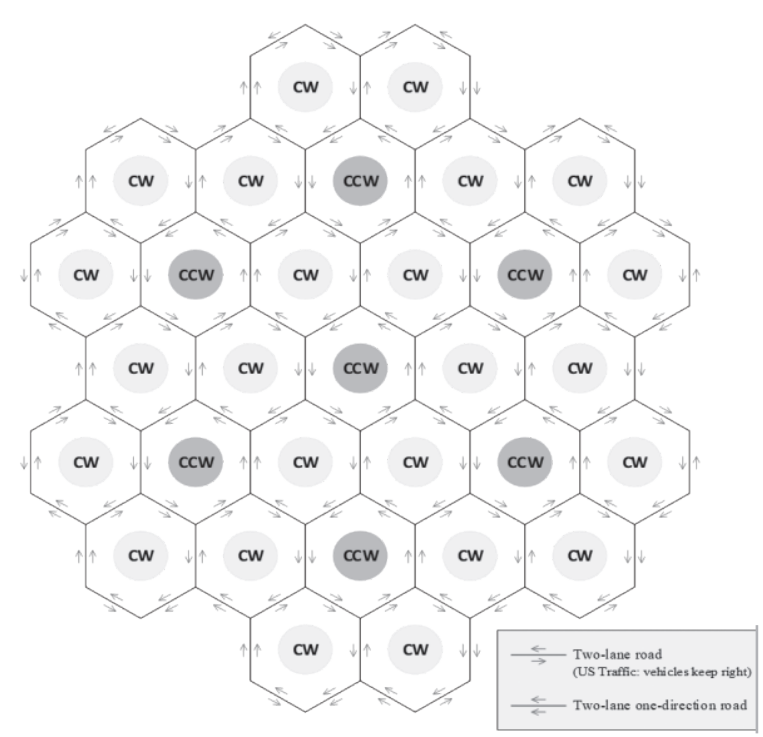

Figure 1 - The Chet Network.

Note:

CW is clockwise traffic circulation.

$\mathrm{CCW}$ is counter-clockwise traffic circulation.

The focus of this research is to move one step further in supporting the design of the Chet network. This research focuses on obtaining the optimum network block size (in terms of block length) of the Chet network, by conducting several tests through changing the block length along with the number of layers and the demand intensity.

\section{Evaluation Parameters}

\subsection{Block Length}

As this research focuses on the optimal block length (hexagon side length) of the Chet network that enhances the network performance, the most important parameter to be tested is the block length. The optimal block length can be obtained by testing different block lengths, then analyzing and comparing the overall network performances of each test case. As a base case, 100-meter is considered as a starting block length. The next test block length is decided after analyzing network performances of the 100-m block length. Result analysis of the first case leads to the next test case. This process continues until it converges.

In summary, $100-\mathrm{m}, 200-\mathrm{m}$, and 300-m are tested. Since the case of $300-\mathrm{m}$ resulted in a congested network, lengths greater than 300-m are not considered. Additionally, the 50-m block length is trialed to compare it with the network performance of the 100-m case. More details about the analysis are explained in the results and discussion section.

\subsection{Number of Lanes}

As shown in Figure 1, each road segment has two lanes. Some segments are one-way (two lanes in one direction) and some are two-way (one lane per direction). They are present in a systematic manner; i.e., the segment between a counterclockwise block and a clockwise block is one-way and the segment between two adjacent clockwise blocks is two-way.

\subsection{Number of Layers}

The Chet network is systematically constructed by layers of counterclockwise (or clockwise) blocks as illustrated in Figure 1. In this study, a different number of layers is also considered. The first layer has only one block (the counterclockwise block in the middle of Figure 1. The second layer adds six clockwise blocks (CW in Figure 1) to the first layer. The network with only one or two layers are considered too small and therefore neglected for simulation.

The third layer adds another six counterclockwise blocks (CCW in Figure 1) to the second layer, and so on. In this study, the Chet network with 3, 4, and 5 layers will be tested to demonstrate the trend. In addition, the 10-layer network is also tested to represent a larger network. The specific configurations of layers are illustrated in Table 1. 
4.4 Network Configurations of Land Use. The configuration of land use is simplified to be two types as explained earlier; i.e., commercial (C) and residential (R) zones. Each network with a different number of layers results in a different value of $C / R$ ratio. The $C / R$ ratios of each network configuration are in Table 1.

Table 1 - Network Configuration Details

\begin{tabular}{|c|c|}
\hline \multirow{3}{*}{$\begin{array}{ll} & \text { Configuration } \\
1 & \end{array}$} & Details \\
\hline & 3-layers \\
\hline & $\begin{array}{c}\text { Total number of } \\
\text { blocks/hexagons - 13 } \\
\text { Number of commercial } \\
\text { (attraction) zones (C)-7 } \\
\text { Number of residential } \\
\text { (distribute) zones (R)-6 } \\
\text { C/R }=7 / 6=\mathbf{1 . 1 6}\end{array}$ \\
\hline 2 & 4-layers \\
\hline & $\begin{array}{c}\text { Total number of } \\
\text { blocks/hexagons - } \mathbf{1 9} \\
\text { Number of commercial } \\
\text { (attraction) zones (C)-7 } \\
\text { Number of residential } \\
\text { (distribute) zones (R)-12 } \\
\mathrm{C} / \mathrm{R}=7 / 12=\mathbf{0 . 5 8}\end{array}$ \\
\hline 3 & \begin{tabular}{|l|} 
5-layers \\
\end{tabular} \\
\hline & $\begin{array}{c}\text { Total number of } \\
\text { blocks/hexagons - } \mathbf{3 1} \\
\text { Number of commercial } \\
\text { (attraction) zones (C)-7 } \\
\text { Number of residential } \\
\text { (distribute) zones (R)-24 } \\
\text { C/R }=7 / 24=\mathbf{0 . 2 9}\end{array}$ \\
\hline 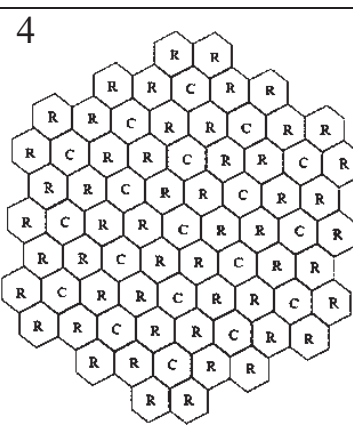 & $\begin{array}{c}\text { 10-layers } \\
\text { Total number of } \\
\text { blocks/hexagons - 73 } \\
\text { Number of commercial } \\
\text { (attraction) zones (C)-19 } \\
\text { Number of residential } \\
\text { (distribute) zones (R)-54 } \\
\mathrm{C} / \mathrm{R}=19 / 54=\mathbf{0 . 3 5}\end{array}$ \\
\hline
\end{tabular}

\subsection{Network Demand}

The Institute of Transportation Engineers, ITE [17] trip generation rates are used to synthesize trip productions and trip attractions at each zone for the AM peak hour and distributed amongst zones all over the network following the gravity model. Different block lengths are tested. As shown in Table 2, the total network demand is the same for different network configurations, given the same block length. For example, with the block length of 100 meters, the total demand used for all configurations are 2,772 vehicles/hour as shown in Table 2.

When changing the block length, the total demand is also changed. As illustrated in Figure 2 , by changing the block length from 100 to 200 meters, the total demand is changed from 2,772 to 11,088 vehicles/hour. Here the implicit assumption is the demand per unit block area is a constant, which is called the density ratio. The same density ratio of 0.107 will be used to derive the total demand for each testing cases as summarized in Table 3.

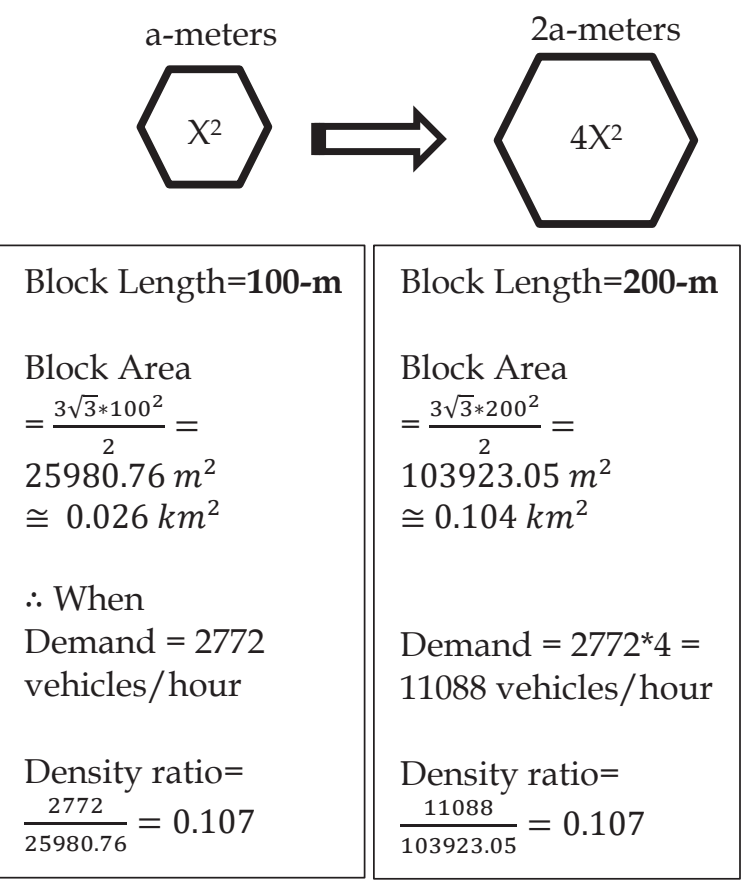

Figure 2 - Total network demand conversion

Primarily, there are two kinds of trips per each block; i.e., entering and exiting trips. For a residential zone (in fact it can be any kind of zones; however, the residential zone is used for illustration) during the AM peak hour, according to ITE [17], the average trip rate is 0.764 trips per dwelling unit with the directional distribution of $16 \%$ entering and $84 \%$ exiting trips.

The base network has 100 meters of block length and 4 layers (Configuration 2 in Table 1). As shown in Figure 2, the block area of the base network is about $0.026 \mathrm{~km}^{2}$. The average house/dwelling size in the US in the year 2010 is 2400 sq. ft. (223 sq. m) according to the Federal Highway Administration, US-DOT [10]. Therefore, the average dwelling units per block are 100. Applying the ITE [17] trip generation rate, the total AM trips per block is, therefore, 77 $\left(0.764^{*} 100\right)$ trips. The exiting trips (trip production) are more reliable (Ortúzar and Willumsen, 2011). Therefore, for a residential 
block, the total trip attractions (AM) is $16 \% * 77=$ 12 trips and trip productions (AM) 84\%*77 = 65 trips.

In the base network, there are 19 blocks in total. Among those, there are 7 commercial blocks and 12 residential blocks. An assumption made here is that the total trips produced from the residential zones are attracted by commercial zones. Therefore, the number of trips attracted by a commercial zone is 111.429 vehicles per hour, which is resulted from $\left(65^{*} 12\right) / 7$. Similarly, the number of trips produced from a commercial zone is 20.571 vehicles per hour, which is a result of $\left(12^{*} 12\right) / 7$. Therefore, the total demand is of 924 vehicles per hour $\left(65^{*} 12+\right.$ $20.571 * 7$ or $111.429 * 7+12 * 12)$. This is considered as the low demand level for the network of the 100-meter block length as indicated in Table 3.

After synthesizing trip productions and trip attractions, the gravity model [21] was used to distribute trips among zones. The generalized travel cost function was assumed to be in the exponential form as shown in the equation.

Where;

$$
f\left(c_{i j}\right)=e^{-\beta c_{i j}}
$$

$c_{i j}$ is the Euclidean distance (displacement) between origin zone $i$ and destination zone $j$

$\beta$ is the cost sensitivity parameter.

The value of 0.1 was assumed for $\beta$ in every testing cases.

In this study, the travel demand is synthesized in such a way that each network is fairly utilized (trips are spreading all over the network per the gravity model) to have a fair comparison among testing cases. To make the experiment more meaningful, the origin-destination matrices of the four configurations are proportionally adjusted to three levels of congestion: (a) low- $x$, (b) medium-3x, and (c) high- $5 x$ travel demands. For example, Table 2 illustrates travel demand patterns of the four configurations of $100-\mathrm{m}$ block length under the medium level of demand; i.e., 2,772 vehicles/hr. The thickness of the lines indicates origin-destination flow. It can be noticed that the travel demands symmetrically distribute along the entire network, because of the gravity model. Therefore, network performances can be quantified to make a fair comparison. For examples, the total demands used in 100-m block length for the cases of low, medium, and high are 924, 2772, and 4620 vehicles per hour, respectively. The rest of the total demands of the other block lengths is obtained by equalizing the ratio of demand per block area (the same density ratio of 0.107 ) as indicated in Table 3.

Table 2 - Trip Desire Lines of Each Network
Configuration

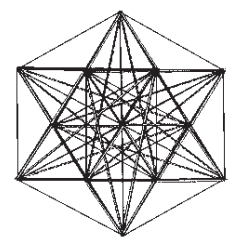

\section{Configuration 1}

Total demand

$=2,772$ vehicles $/$ hour

Total displacement

$=897.46$ vehicle-

kilometres

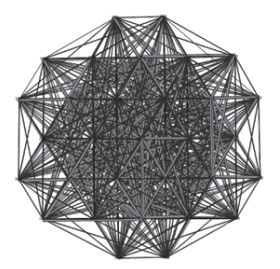

\section{Configuration 3}

Total demand

$=2,772$ vehicles $/$ hour

Total displacement

$=1,260.30$ vehicle-

kilometres

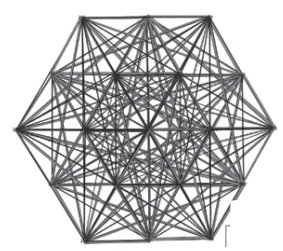

Configuration 2

Total demand

$=2,772$ vehicles $/$ hour

Total displacement

$=1,055.57$ vehiclekilometres

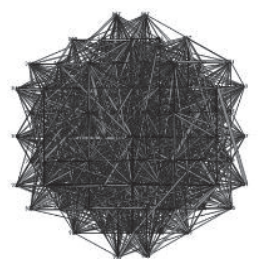

Configuration 4

Total demand

$=2,772$ vehicles $/$ hour

Total displacement

$=1968.31$ vehicle-

kilometres

\section{Network Details}

Traffic demand is loaded to each network using balanced origin-destination matrix. Centroids are used to serve as origins and destinations. There are six separated links (centroid
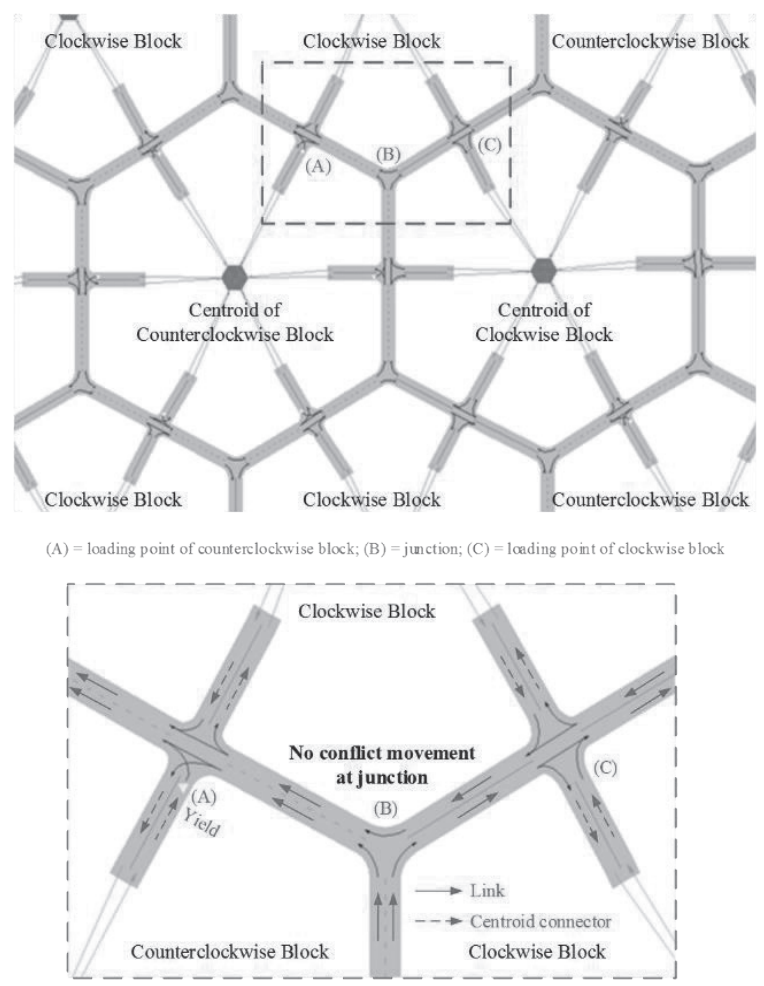

Figure 3 - Network Coding in TransModeler 
connectors) to transfer trips from the centroid to major traffic stream. Figure 3 graphically illustrates network coding in TransModeler. Explanation that is more detailed can be found in [23].

\section{$5.1 \quad$ Testing Cases}

In order to determine the optimum block length, a series of testing cases are fairly defined and analysed. Three demand levels with four types of block lengths and four network configurations are considered; and under each demand, there are 16 test cases, therefore, in total 48 test cases. The total demand for each test case is shown in Table 3.

Table 3 - Total Demand for Testing Cases

\begin{tabular}{|l|c|c|c|c|}
\hline $\begin{array}{l}\text { Total Demand (given } \\
\text { the same density } \\
\text { ratio of 0.107) }\end{array}$ & 50-meter & 100-meter & 200-meter & 300-meter \\
\cline { 2 - 5 } & \multicolumn{4}{|c|}{2 lanes } \\
\hline Block area & $0.006 \mathrm{Km}^{2}$ & $0.026 \mathrm{Km}^{2}$ & $0.104 \mathrm{Km}^{2}$ & $0.234 \mathrm{Km}^{2}$ \\
\hline $\begin{array}{l}\text { Low Demand (x) } \\
\text { veh/hr }\end{array}$ & 231 & 924 & 3696 & 8316 \\
\hline $\begin{array}{l}\text { Medium Demand } \\
\text { (3x) veh/hr }\end{array}$ & 693 & 2772 & 11088 & 24948 \\
\hline $\begin{array}{l}\text { High Demand (5x) } \\
\text { veh/hr }\end{array}$ & 1155 & 4620 & 18480 & 41580 \\
\hline
\end{tabular}

\subsection{Microscopic Traffic Simulator}

In this study, TransModeler (version 4.0) is used as the microscopic traffic simulator. TransModeler simulates how a vehicle reacts with surrounding vehicles and infrastructures. The processes includes car following and lane changing, merging and weaving behaviours, etc. The option of stochastic shortest path is used for the route choice this study. According to Caliper [3], every path cost is randomized and every vehicle in the network accounts for different variations in perception and behaviours. Each one-hour simulation, which represents the morning (AM) peak hour, is run for five times for each testing case. Variations of performance indicators are checked and the median run out of the five is selected to represent the testing case. The 48 testing cases are coded as summarized in Table 3. Each individual vehicle is generated from the centroid of its origin zone and moves to the centroid of its destination zone.

In this study, all the road segments were coded with a capacity of $1200 \mathrm{pc} / \mathrm{h} / \mathrm{ln}$, the free flow speed of $56.3 \mathrm{~km} / \mathrm{hr}$ and a speed limit of 40.2 $\mathrm{km} / \mathrm{hr}$. This is consistent with most of the urban streets in the US where the speed limit for urban or residential areas is set at 20-30 miles per hour (32-48 kilometres/hour) as documented in [19].

\section{Results and Discussions}

Each testing case is analysed and compared with the others by using four key performance indicators at the network level, which are extracted from the microscopic traffic simulator.

\subsection{Average Speed $(\mathrm{km} / \mathrm{h})$}

The average speed here is the travel speed averaged over every vehicle that completed their trips within the simulation period. There are some vehicles still in the network when the run ends in some testing cases; these vehicles were excluded from the average speed computation. This can be considered as one of the main indicators that can compare various cases with minimal bias, as it is weighted by the number of completed vehicles.

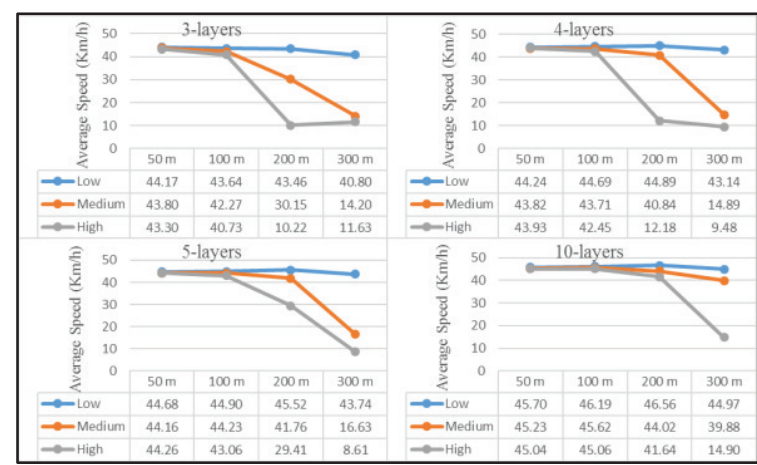

Figure 4 - Graphical Illustration: Avg. Speed

Figure 4 graphically illustrates the average speeds of each testing cases. It can be seen that the 50-meter and 100-meter block lengths of the Chet network being equally superior to the other two block lengths as it performs the highest average network speed at every demand level. The network of 200-m block length performs well for low and medium demand.

\subsection{Average Delay (seconds/vehicle)}

The average delay is the delay experienced on the link, averaged over all the vehicles that travelled on the link during the interval.

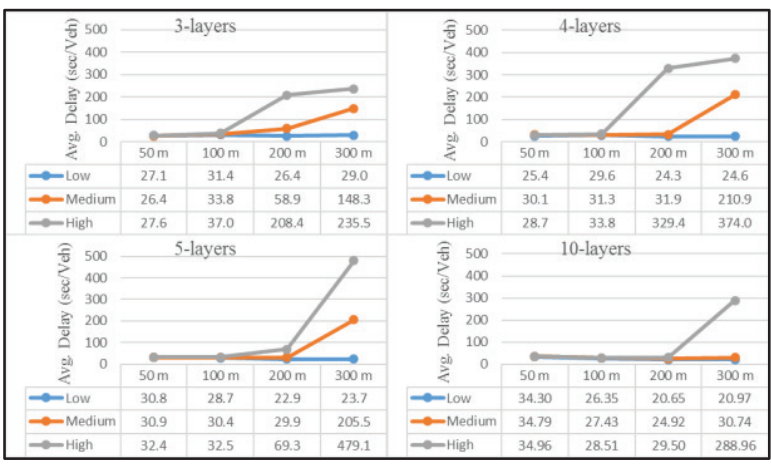

Figure 5 - Graphical Illustration: Avg. Delay 
50-m and 100-m block lengths of the Chet network is again being superior as it performs the lowest total network delay at every demand level according to the results shown in Figure 5. In addition, the 200-m network performs equally well for low and medium demand levels.

\subsection{Stopped Time (Hours)}

Stopped time here is the summation of total stopped time experienced by all vehicles that completed their trips within the simulation period.

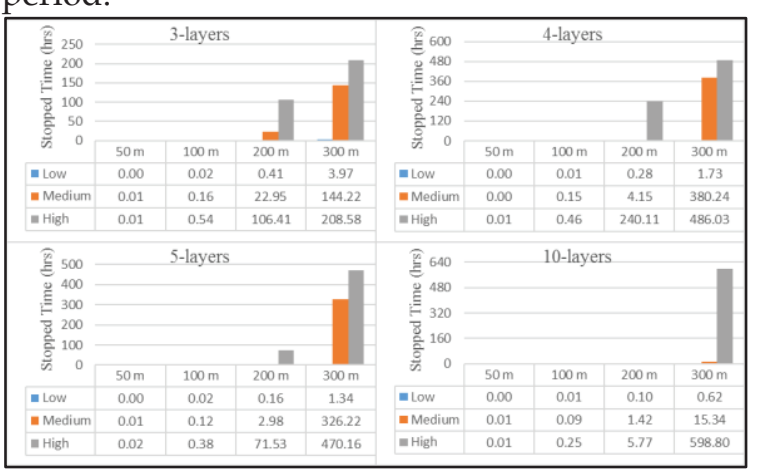

Figure 6 - Graphical Illustration: Stopped Time

In summary, 50-m, 100-m and 200-m block lengths of Chet transport network being superior as it performs the lowest stopped time at low and medium demand levels according to Figure 6. At high demand level, 50-m and 100-m block lengths still perform well while cases in $200-\mathrm{m}$ and $300-\mathrm{m}$ block lengths result in a significant increment in the stopped time.

\subsection{Trips Completion Rate (Percentage)}

The completed trips are the total completed trips during the simulation interval and trips that are en-route, yet not arrived or waiting to enter the network by simulation ends are called incompleted trips. Therefore, the percentage of completed trips out of total trips during the simulation period is called trip completion rate in percentage. If it is significantly less than $100 \%$, gridlocks may happen and could bias the comparisons.

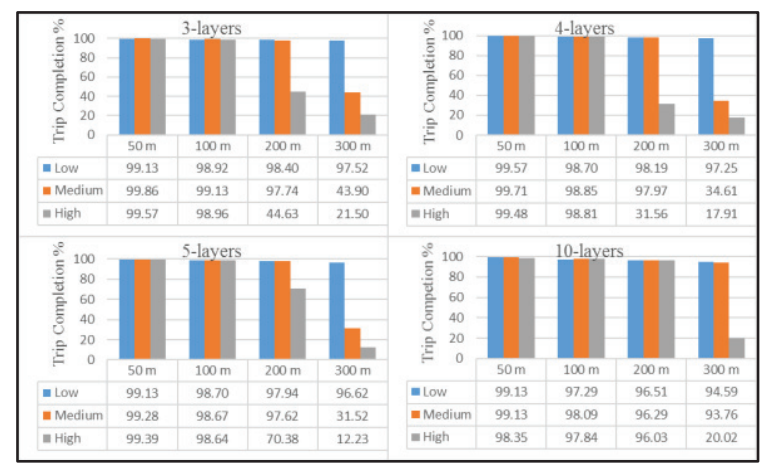

Figure 7 - Graphical Illustration: Trips Completion Rate (\%)
From Figure 7, it can be seen that the 50-m and 100 -m block lengths of the Chet network is being superior as it results in the highest trip completion rate at every demand level. In addition, except for high demand 200-m performs equally well for low and medium demand levels. When the network is large, vehicles should travel farther, hence those larger networks (10-layers) results in lesser trip completion rate comparing to smaller networks. Also, larger networks are well distributed, so less congested, hence 2-lanes of larger block lengths in larger networks is better than in smaller networks.

\section{Conclusions}

This study explores the Chet network by focusing on the optimal configuration in terms of the block size. Several testing cases were defined by varying the block length $(50,100,200$ and 300 meters) along with the number of layers $(3,4,5$ and 10 layers) and the demand intensity (low, medium and high).

The Chet network (as illustrated in Figure 1) composes of hexagons tiling together to form a unique, novel design of directional links to avoid conflict movements at junctions. There are several advantages to using this network, which can be summarized as follows.

1. No conflict movement at junctions; vehicles can move from one place to another without any unnecessary stop.

2. The Chet network is economical, as it requires a shorter road length to cover a certain area when compared with the grid network.

3. The 120-degree turn in the Chet network is safer and more efficient than the 90-degree turn in the grid network.

Even though major delays due to primary conflicts at junctions were, completely removed, minor delays due to lane changing along the road still exist. Therefore, a microscopic traffic simulator (TransModeler) was used to conduct several testing cases in obtaining the optimum block length for the Chet network.

The 48 testing cases were defined and simulated to compare the overall performances. The optimum block length can be obtained by analysing simulation results that lead to the highest efficiency in terms of average speed, average delay, stopped time and trips completion rate. Realistic sets of travel demands were assumed by applying the ITE trip 
generation rates [17] of the morning peak. In order to fairly compare the results of different block lengths (50, 100, 200, and 300 meters), the number of trips per block area is assumed to be constant (same density ratio as shown by an example in Figure 2). As a result, the total demands of each block length set of experiments are not equal. In addition, the levels of demand (low, medium and high) was also tested (as summarized in Table 3). In all testing cases, the demands are spreading all over the network with the same gravity model to have a fair comparison.

\subsection{Key Findings}

The results of every key performance indicator evident that the Chet network with 50-m to 100$\mathrm{m}$ is remarkable. In addition, $100-\mathrm{m}$ to the $200-\mathrm{m}$ range is acceptable at low (normal) and medium (3 times higher than normal) levels of demand. The numerical results suggest the optimal block length around 100 meters. Performance reductions relative to the optimal one are observed in the cases 200 and 300 meters and expected from the trend in the cases of larger block lengths. That is because the same density ratio (trips per area) is used; the larger the block length, the higher the number of trips to and from each block. As a result, in the case of the very large block length, the demand will be very high and can cause gridlock due to link capacity.

In summary, the Chet network with 100-meter block length (hexagon side) is optimal and robust. It performs better than the longer block lengths under every demand level. On the other hand, for the shorter block length (e.g. 50 meters); only small benefits are gained in terms of network performances. However, it will be costly in construction and maintenance.

The Chet network is more suitable for a new area of built environment than for replacing the existing network. The fascinating appearance and the simulation results are encouraging. However, prior to a real implementation, there are several other issues beyond the scope of this study to be considered. Some further research possibilities are suggested in the following section.

\subsection{Further Research Possibilities}

This section recommends some potential future researches, which may lead to the future real-life implementation of the Chet network.

- It is interesting to compare the Chet network with an existing equivalent city area to create a more realistic case study.
- In this study, the gravity model was used to distribute trips all over the network in order to make a fair comparison among several testing cases. However, in the real world, it might not be the case. Therefore, analysing the robustness of network performance under various demand patterns is also interesting.

- The proposed network may significantly gain mobility benefit but could cause traveller confusion in wayfinding. A navigation system could be invented, including zonal numbering system and guide signs to help travellers navigating from one place to another.

- In this study, the focused mode of transportation is the car. It is interesting to explore how to handle pedestrians and bicyclists.

- This network could be used in other applications in addition to urban streets for cars. Possible applications could be an atgrade escalator network for walkers in airports or shopping malls, a canal network for boats, and a walking street network for shoppers, a transit network for vehicles that can make a 120-degree turn, a manufacturing line in factories, etc.

\section{Acknowledgment}

The authors wish to thank the Asian Institute of Technology (AIT) for the research initiation grant that encouraged and supported to the success of this research. Furthermore, heartfelt acknowledge for the assistance given by the IESL in publishing this research.

\section{References}

1. Bally, D.C., 2010. Thinking outside the blocks - exploring alternatives to traditional neighborhood design. ESRI International User Conference.

2. Ben-Joseph, E., Gordon, D., 2000. Hexagonal planning in theory and practice. Journal of Urban Design, 5 (3), 237-265.

3. Caliper, 2015. TransModeler User's Guide. Caliper Corporation, MA.

4. Cauchon, N., 1925. Hexagonal blocks for residential districts. The American City, 17, 145-146.

5. Cauchon, N., 1927. Planning organic cities to obviate congestion, orbiting traffic by hexagonal planning and interceptors. Annals of the American Academy of Political and Social Science, 133, 241-246. 
6. Cauchon, N., 1929. The six-sided block. National Real Estate Journal, 30, 42-46.

7. Dotoli, M., Fanti, P., Meloni, C., 2004. Coordination and real-time optimization of signal timing plans for urban traffic control. Proceedings of the 2004 IEEE International Conference on Networking, Sensing and Control, Taipei, Taiwan.

8. Eichler, D., Bar-Gera, H., Blachman, M., 2013. Vortex-based zero-conflict design of urban road networks. Networks and Spatial Economics, 13(3), 229-254.

9. Fan, L., 2014. Coordinated control of traffic signals for multiple intersections. Applied Mathematics, 5, 2042-2049.

10. Federal Highway Administration, USDOT, 2010 https://www.fhwa.dot.gov/ Accessed July 24, 2016.

11. FHWA, 2009. Manual on uniform traffic control devices for streets and highways (MUTCD). Federal Highway Administration, 2009 Edition.

12. Girianna, M., Benekohal, R., 2003. Signal coordination for a two-way street network with oversaturated intersections. Proceeding of the 82nd Transportation Research Board Annual Meeting, Washington D.C.

13. Grammenos, F., Pogharian, S., TaskerBrown, J.,2002. Residential street pattern design.Socio-economic Series75.

14. Hales, T.C., 2001. The honeycomb conjecture. Discrete and Computational Geometry, 25 (1), 1-22.

15. Han, K., Gayah, V., 2015. Continuum signalized junction model for dynamic traffic networks: offset, spillback, and multiple signal phases. Transportation Research Part B, 77, 213-239.

16. Highway Capacity Manual, 2010.

17. ITE, 2008. Trip Generation. Institute of Transportation Engineers, 8th Edition, ISBN-13:978-1-933452-43-2.

18. Melut, P. and O'Sullivan, P., 1974. A comparison of simple lattice transport networks for a uniform plain. Geographical Analysis, 6(2),pp.163-173.

19. New York City, Department of

Transportation.

http://www.nyc.gov/html/dot/html/ motorist/motorist.shtml_Accessed

August 14, 2016.

20. Omolo-Okalebo, F., Sengendo, H., 2011. Perspectives on City Planning of PostIndependence Kampala: The Emergence of the Metropolitan Growth Model and the Hexagonal Cell.
21. Ortúzar, J.D., Willumsen, L.G., 2011. Modelling Transport. John Wiley \& Sons, Ltd., ISBN 9780470760390.

22. Pillai, R., Rathi, A., Cohen, S., 1998. A restricted branch-and-bound approach for generating maximum bandwidth signal timing plans for traffic networks. Transportation Research Part B, 32 (8), 517-529.

23. Pravinvongvuth S., 2016. A new atgrade transportation network without signalized intersection, roundabout, or stop sign for land use and transportation planning. World Conference on Transport Research WCTR 2016 Shanghai.

24. Price,A.,2013.Optimizing the StreetGrid http:/ / www.strongtowns.org/journal /2013/11/27/optimizing-the-streetgrid.html\#.U_6_nNKSxg- Accessed October 17, 2016.

25. Wong, S., 1996. Group-based optimization of signal timings using the TRANSYTtrafficmodel. Transportation Research Part B, 30 (3), 217-244. 